\title{
Positivity constraints for Off-Forward Parton Distributions
}

\author{
B. Pire ${ }^{a}$, J. Soffer ${ }^{b}$ and O.Teryaev ${ }^{a, c}$ \\ ${ }^{a} \mathrm{CPhT}$, Ecole Polytechnique, F-91128 Palaiseau, France \\ ${ }^{b}$ CPT-CNRSF, F-13288 Marseille Cedex 9, France \\ ${ }^{c}$ Bogoliubov Laboratory of Theoretical Physics, \\ Joint Institute for Nuclear Research \\ 141980 Dubna, Moscow region, Russia]
}

\begin{abstract}
Off-Forward Parton Distributions (OFPD's) are new hadronic objects which may be measured in various exclusive reactions. We derive non-trivial positivity constraints for them that should allow to get extra restrictions for model inputs.
\end{abstract}

PACS numbers:12.38.Bx, 13.88.+e

Unité Propre de Recherche 7061

CPT-98/P.3633

Web address: www.cpt.univ-mrs.fr

\footnotetext{
${ }^{1}$ Unité Mixte de Recherche C7644 du Centre National de la Recherche Scientifique

${ }^{2}$ Unité Propre de Recherche 7061 du Centre National de la Recherche Scientifique

${ }^{3}$ Permanent address
} 
1 - The concept of Off-Forward (non-forward, non-diagonal) Parton Distributions (OFPD), related to the matrix elements of non-local string operators [1], has attracted much attention since it has been recognized [2, 3] that these new objects describing the deep hadronic structure could be measured in deep exclusive reactions such as forward virtual Compton scattering and diffractive electroproduction of mesons. They factorize 3, 14 from a hard subprocess amplitude, provided the virtuality $Q^{2}$ of the photon is large enough for the differential cross section to enter a scaling regime, where the handbag type diagrams dominate[5]. As for any long distance dominated object, not much is known about these distributions, except some limiting values obtained from already measured standard forward parton distributions. Various model estimates have been recently proposed [2, 6, 0, 8, 9], but in the absence of any trustable non-perturbative QCD calculations, we want here to advocate the usefulness of bounds coming from positivity requirements for constructing models, which should allow to get seriously guided rate estimates for several proposed experiments at CEBAF, CERN and DESY.

For every parton species, there are six off-forward parton distributions. They all depend on three kinematical variables, which can be chosen as $x$, the light-cone fraction of the parton emitted by the proton target, $x^{\prime}$, the fraction of the parton absorbed by the scattered proton, and $t$, the momentum transfer between the initial and final proton. Both momentum fractions are measured with respect to the initial proton momentum $p$ [3]. It is meaningful for positivity studies as well as for symmetry properties [10] to reexpress the light-cone fraction of the parton absorbed by the final proton as a fraction with respect to that proton's momentum, i.e. $x_{1}=x$ and $x_{2}=x^{\prime} /\left(1-x+x^{\prime}\right)$. This may be compared with the symmetric choice [2], which, strictly speaking, is understood when OFPD are considered. We restrict ourselves to the case when the momentum fraction of the second (absorbed) parton is positive, since there is then a clear relation between the OFPD and ordinary distribution functions measured in deep inelastic scattering [9]. Kinematics fixes $t$ and the difference $x-x^{\prime}$ to some fixed value like $x_{b j}$ in the deeply virtual Compton scattering (DVCS) process, while the scattering amplitude has an imaginary part with $x^{\prime}=0$ and a real part, which is a principal part 
integral over $x^{\prime}$. Here we pay special attention to spin-averaged quark $q_{\zeta}(x)$ [3] and gluon $g\left(x, x^{\prime}, t\right)$ [8] distributions.

The $t$-dependence of the OFPD's is governed by the proton form factors through relations such as : $\int_{-1}^{+1} d x_{1} g\left(x_{1}, x_{2}, t\right)=F(t)$. It is reasonable to assume that this $t$-dependence factors out. Remember however that kinematics fixes $t_{\text {min }} \neq 0$. The OFPD's acquire a $Q^{2}$-dependence governed by evolution equations 22, 3, 10, 11, 12, and we show at the end of the paper that the QCD $Q^{2}$ - evolution preserves the validity of the positivity bound.

2 - Since our present knowledge on OFPD's is rather limited, any rigorous bounds for them are of great interest. The aim of the present paper is to develop such bounds, coming from positivity of the density matrix. Because the OFPD's do not have a probabilistic interpretation, one may wonder if this is possible at all. However, non-diagonal elements of a density matrix are constrained by positivity as well as its diagonal elements, as shown by the Soffer bound on the chiral-odd quark distribution $h_{1}^{q}(x)$ [13] (this distribution is forward in momentum, but it is non-diagonal in helicity), which reads [14]:

$$
\left|h_{1}^{q}(x)\right| \leq q_{+}(x) \equiv \frac{1}{2}[q(x)+\Delta q(x)],
$$

where $q$ and $\Delta q$ are the usual spin-averaged and spin-dependent quark distributions. Although this interesting result was originally proven at the level of the parton model, it was shown recently that it is preserved by the QCD $Q^{2}$ evolution, up to next-to-leading order 15, 16].

The OFPD's are elements of parton density matrices which are nondiagonal in momentum, and they may be treated in a similar way, provided the momentum fraction of the absorbed parton is positive[17]. Moreover, in the recent paper $[8]$ the inequality

$$
2 x^{\prime} g\left(x, x^{\prime}\right) \leq x g(x)+x^{\prime} g\left(x^{\prime}\right)
$$

was obtained by a rather similar method to that of 14 .

Let us now derive another, stronger (especially at low $x$ ) inequality, and outline the method allowing to derive similar inequalities for the various spin 
components of the OFPD's. We present here the derivation with some details, in order to stress the dependence of the actual definition of the non-forward distribution 7 .

Let us start the discussion with the simpler case of non-polarized quark distribution, which by introducing the light-cone decomposition of the quark fields [18] is analogous to the scalar one. The quark forward distribution is just

$$
\begin{gathered}
q(x)=\int \frac{d \lambda}{4 \pi} e^{i \lambda x}<p, S|\bar{\psi}(0) h \psi(\lambda n)| p, S>= \\
\frac{1}{\sqrt{2} p^{+}} \int \frac{d \lambda}{2 \pi} e^{i \lambda x}<p, S\left|\phi^{+}(0) \phi(\lambda n)\right| p, S>,
\end{gathered}
$$

where $\phi$ is the good component of the quark field and the light-cone vectors are normalized such as $p n=p^{+} n^{-}=1$. By inserting a complete set of intermediate states $\mid X>$ and making use of the generalized optical theorem and the fact that the matrix elements may be replaced by their imaginary parts [17], the forward distribution can be written as

$$
q(x)=\sum_{X} \frac{1}{\sqrt{2} p^{+}}|<p, S| \phi(0)|X>|^{2} \delta\left(x-\left(p-p_{X}\right) n\right) .
$$

The quark non-forward distribution reads,

$$
q_{\zeta}(x)=\frac{1}{\sqrt{1-\zeta}} \int \frac{d \lambda}{4 \pi} e^{i \lambda x}<p, S|\bar{\psi}(0) \not h \psi(\lambda n)| p^{\prime}, S>,
$$

where the factor $\sqrt{1-\zeta}$ comes from the bilinear $\bar{u}\left(p^{\prime}\right) u(p)$, in the definition. By an analogous procedure as above, it becomes

$$
\begin{array}{r}
q_{\zeta}(x)=2 \operatorname{Re} \sum_{X} \frac{1}{\sqrt{2(1-\zeta)} p^{+}}<p, S|\phi(0)| X> \\
<p^{\prime}, S|\phi(0)| X>^{*} \delta\left(x-\left(p-p_{X}\right) n\right),
\end{array}
$$

\footnotetext{
${ }^{4}$ We are indebted to A.V. Radyushkin for pointing out the correct definitions and for helpful comments, which allowed to restore the related factors, which were missing in the original version of this paper.
} 
where we used the hermiticity of the matrix element. We are now ready to write down the Cauchy-Schwarz inequality as:

$$
\sum_{X}|<p, S| \phi(0)\left|X> \pm a<p^{\prime}, S\right| \phi(0)|X>|^{2} \delta\left(x-\left(p-p_{X}\right) n\right) \geq 0,
$$

where $a$ is a positive number, which we put equal to 1 for the time being. While the non-diagonal term of (7) is producing just the non-forward distribution, and the first diagonal term - the distribution $q(x)$, the second diagonal term should be studied in more details:

$$
\begin{array}{r}
\sum_{X} \frac{1}{\sqrt{2} p^{+}}\left|<p^{\prime}, S\right| \phi(0)|X>|^{2} \delta\left(x-\left(p-p_{X}\right) n\right)= \\
\sum_{X} \frac{1}{\sqrt{2} p^{+}}\left|<p^{\prime}, S\right| \phi(0)|X>|^{2} \delta\left(x^{\prime}-\left(p^{\prime}-p_{X}\right) n\right)= \\
\sum_{X} \frac{1}{\sqrt{2} p^{\prime}+}\left|<p^{\prime}, S\right| \phi(0)|X>|^{2} \delta\left[x^{\prime}\left(n^{\prime-} / n^{-}\right)-\left(p^{\prime}-p_{X}\right) n^{\prime}\right]=q\left(x_{2}\right) .
\end{array}
$$

Here the necessary rescaling of the light-cone coordinate, which is required to get the definition (31) is making the argument equal just $x_{2}$, while the overall factor $1-\zeta$ coming from the rescaling of the delta-function argument, is precisely cancelled with the rescaling of the factor $1 / p^{+}$(which is the natural consequence of the correct transformation properties of $q(x)$ ), so that the overall rescaling of the diagonal term [9] is actually manifested for the scalar case only.

As a result, we have the following inequality

$$
\left|q_{\zeta}(x)\right| \leq \frac{1}{\sqrt{1-\zeta}}\left[q\left(x_{1}\right)+q\left(x_{2}\right)\right]
$$

for the spinor case, and

$$
\left|q_{\zeta}(x)\right| \leq q\left(x_{1}\right)+\frac{1}{1-\zeta} q\left(x_{2}\right)
$$


for the scalar case. By restoring the dependence on the parameter $a$, one is led to

$$
\left|q_{\zeta}(x)\right| \leq \frac{1}{\sqrt{1-\zeta}}\left[a q\left(x_{1}\right)+\frac{1}{a} q\left(x_{2}\right)\right]
$$

for spinor quarks and to

$$
\left|q_{\zeta}(x)\right| \leq a q\left(x_{1}\right)+\frac{1}{a(1-\zeta)} q\left(x_{2}\right)
$$

for scalar quarks. By minimizing the r.h.s. with respect to the variation of $a$, we finally get 9

$$
\left|q_{\zeta}(x)\right| \leq \sqrt{\frac{q\left(x_{1}\right) q\left(x_{2}\right)}{1-\zeta}},
$$

for both scalar and spinor quarks. A similar bound can be obtained for Ji's off-forward quark distribution, that is

$$
\left|H_{q}(x, \xi)\right| \leq \sqrt{\frac{q\left(x_{1}\right) q\left(x_{2}\right)}{1-\xi^{2}}},
$$

where $x_{1,2}=(x \pm \xi) /(1+\xi), \xi=\zeta /(2-\zeta)$.

The derivation for the gluons is analogous. The forward and nonforward [3], 8] distributions may be expressed as

$$
\begin{array}{r}
x g(x)=\frac{1}{2} \sum_{X, i}|<p, S| G^{+i}(0)|X>|^{2} \delta\left(x-\left(p-p_{X}\right) n\right), \\
x_{2} g\left(x_{2}\right)=\frac{1}{2(1-\zeta)} \sum_{X, i}\left|<p^{\prime}, S\right| G^{+i}(0)|X>|^{2} \delta\left(x-\left(p-p_{X}\right) n\right), \\
x^{\prime} g\left(x_{1}, x_{2}\right)=\frac{1}{\sqrt{1-\zeta}} R e \sum_{X}<p, S\left|G^{+i}(0)\right| X> \\
<p^{\prime}, S\left|G^{+i}(0)\right| X>^{*} \delta\left(x-\left(p-p_{X}\right) n\right),
\end{array}
$$

where the summation over $i$ stands to select the transverse components of the gluon field of strength $G$. The Cauchy-Schwarz inequality leads to

$$
\left|x^{\prime} \sqrt{1-\zeta} g\left(x_{1}, x_{2}\right)\right| \leq \frac{1}{2}\left[x_{1} g\left(x_{1}\right)+(1-\zeta) x_{2} g\left(x_{2}\right)\right],
$$


and, after minimization with respect to the variation of $a$, one is led to

$$
\left|x^{\prime} g\left(x_{1}, x_{2}\right)\right| \leq \sqrt{x_{1} x_{2} g\left(x_{1}\right) g\left(x_{2}\right)} .
$$

Equality (16) for $x_{2} \sim x^{\prime} \ll x$ is numerically close to (2). However, the symmetry properties for the variables $x_{1}, x_{2}$ are simpler

$$
x^{\prime}\left(x_{1}, x_{2}\right) g\left(x_{1}, x_{2}\right)=x^{\prime}\left(x_{2}, x_{1}\right) g\left(x_{2}, x_{1}\right) .
$$

It was stressed [9] that in the case of the double distribution this symmetry is manifested, provided the overall factor $1-\zeta / 2$ [10] is extracted. However, the symmetry of nonforward distribution is more complicated in that case and one can see that

$$
F_{\zeta}(X)=\frac{1}{1-\zeta} F_{-\frac{\zeta}{1-\zeta}}\left(\frac{X-\zeta}{1-\zeta}\right) .
$$

Some comments are in order. First, all the considered inequalities are also valid, when the $t$ dependence of the OFPD's is present in the l.h.s., while this dependence is absent in the r.h.s. due to Lorentz invariance (c.f. [19]). Second, the $x$ dependence of the two terms in (9) is not governed by Lorentz invariance, as the light-cone direction is crucial.

3 - Let us now take into account the spin degrees of freedom. To do so, we consider the quantities $\left\langle p, S\left|G^{+i}(0) \pm \tilde{G}^{+i}(0)\right| X>\right.$, corresponding to a definite gluon helicity, while the hadron helicities are fixed to be positive, leading to the absence of the contributions which are non-diagonal in helicity indices. By applying the same method as above, one easily gets

$$
2 x^{\prime} \sqrt{1-\zeta}\left|g^{ \pm}\left(x_{1}, x_{2}\right)\right| \leq x_{1} g^{ \pm}\left(x_{1}\right)+x_{2}(1-\zeta) g^{ \pm}\left(x_{2}\right) .
$$

By adding these two inequalities, one checks that (16) is still valid, so that unpolarized distributions are decoupling from the polarized ones. This is no more valid in the case of the optimized inequalities

$$
x^{\prime}\left|g^{ \pm}\left(x_{1}, x_{2}\right)\right| \leq \sqrt{x_{1} x_{2} g^{ \pm}\left(x_{1}\right) g^{ \pm}\left(x_{2}\right)},
$$


leading to the bound

$$
x^{\prime}\left|g\left(x_{1}, x_{2}\right)\right| \leq \sqrt{x_{1} x_{2} g\left(x_{1}\right) g\left(x_{2}\right)} \cdot \lambda\left[P\left(x_{1}\right), P\left(x_{2}\right)\right],
$$

with $2 \lambda\left[P\left(x_{1}\right), P\left(x_{2}\right)\right]=\sqrt{\left(1+P\left(x_{1}\right)\right)\left(1+P\left(x_{2}\right)\right)}+\sqrt{\left(1-P\left(x_{1}\right)\right)\left(1-P\left(x_{2}\right)\right)}$ , where one introduces the gluon polarization, defined as $P(x)=\Delta G(x) / G(x)$ and such as $|P(x)| \leq 1$. This inequality, in principle, offers a possibility of extracting information on the gluon spin-dependent distribution $\Delta G$ from the unpolarized diffractive processes. Conversely, if one knows $\Delta G$ one gets an inequality which is stronger than (17) since one has always the inequality $\lambda\left[P\left(x_{1}\right), P\left(x_{2}\right)\right] \leq 1$.

The inequality (17) in turn provides a stronger bound on $g\left(x_{1}, x_{2}\right)$, in comparison with (16), and this is related to the difference between $g\left(x_{1}\right)$ and $g\left(x_{2}\right)$. It is especially pronounced when one of the $x$ is small, a situation occuring in diffractive electroproduction. At the same time, a bound for the behaviour of the OFPD's in the quasielastic region $x_{1} \rightarrow 1, x_{2}=$ const. is implied by the stronger inequality (17), while it cannot be derived from the weaker one. Namely, the OFPD's should decrease like $\left(1-x_{1}\right)^{\beta / 2}$, where the power $\beta$ characterizes the decrease of the forward distribution and is related to the form factor behaviour by the quark counting rules. In particular, the ratio $R$ defined in [8], as $x^{\prime} g\left(x, x^{\prime}\right) / x g(x)$ is bounded as

$$
R \leq \sqrt{\frac{x^{\prime} /\left(1-x+x^{\prime}\right) g\left(x^{\prime} /\left(1-x+x^{\prime}\right)\right)}{x g(x)}} \approx \sqrt{\frac{x^{\prime} g\left(x^{\prime}\right)}{x g(x)}},
$$

where we neglected the difference between $x^{\prime}$ and $x_{2}$.

For a better estimate one may use the parametrization $g(x)=$ $N x^{-\alpha}(1-x)^{\beta}$, so that the growth of $\mathrm{R}$ for small $x, x^{\prime}$ is bounded as

$$
R \leq\left(x / x^{\prime}\right)^{\frac{\alpha-1}{2}},
$$

while the power is twice larger for the weaker bound. 
4- Up to now, we have considered non-diagonality either in helicity [14 or in momentum. It is also possible to consider both effects together, by a simple generalization of the outlined method. Let us consider in the quark sector the distributions $q_{+}(x)$ and $h\left(x, x^{\prime}\right)$, the latter being the non-forward generalization of transversity 20]. By optimization with respect to $a$, one gets the obvious non-trivial bound

$$
\left|h\left(x_{1}, x_{2}\right)\right| \leq \sqrt{q_{+}\left(x_{1}\right) q_{+}\left(x_{2}\right)} .
$$

One may derive other inequalities, considering various combinations of the quantities $a$, and varying the helicity indices in their definitions.

5- To check the validity of the positivity bounds in the case of the leading order $Q^{2}$ evolution, one may use the kinetic interpretation of the latter [21, 16], similarly to the proof for the Soffer inequality. As a result, one finds that the positivity constraint $(17)$ is preserved provided the following inequality is satisfied

$$
\sqrt{\frac{z^{\prime}(1-z)}{z\left(1-z^{\prime}\right)}} P\left(z, z^{\prime}\right) \leq \sqrt{P(z) P\left(z^{\prime}\right)},
$$

where $P(z)$ and $P\left(z, z^{\prime}\right)$ are the non-singular parts of the diagonal and offdiagonal [8] splitting kernels. Note that the factor $\sqrt{\frac{z^{\prime}(1-z)}{z\left(1-z^{\prime}\right)}}$ makes the 1.h.s. symmetric with respect to the interchange $z \leftrightarrow z^{\prime}$. Since $P\left(z, z^{\prime}\right)$ is itself symmetric with respect to the transformation $z \rightarrow 1-z^{\prime}, z^{\prime} \rightarrow 1-z$, the l.h.s. is also symmetric with respect to the simultaneous interchange $z \leftrightarrow$ $1-z, z^{\prime} \leftrightarrow 1-z^{\prime}$, while the symmetry with respect to these interchanges made separately, also respected by r.h.s., is violated by the factor $1 / 2$ of the two last terms in $P\left(z, z^{\prime}\right)$. One easily checks that (26) is actually satisfied by the kernel [8]. In the next-to-leading order case, the positivity is dependent on the factorization scheme and may be used [21], as an extra constraint, for making the suitable choice.

In conclusion, let us stress that the positivity constraints derived here will 
help model builders to improve their rate estimates for proposed electroproduction experiments.

Acknowledgements We acknowledge useful discussions and correspondence with M. Diehl, I.V. Musatov, A.V. Radyushkin and J.P. Ralston. This investigation was partially supported by grants 96-02-17361 from Russian Foundation for Fundamental Research and 93-1180ext from INTAS.

\section{References}

[1] B. Geyer, D. Robaschik, M. Bordag and J. Hořejší Z. Phys. C26 (1985) 591; T. Braunschweig, B. Geyer, J. Hořejší and D. Robaschik, Z. Phys. C33 (1987) 275; F.-M. Dittes, D. Müller, D. Robaschik and B. Geyer, Phys. Lett. B209 (1988) 325; I.I. Balitskii and V.M. Braun, Nucl. Phys. B311 (1988/89) 541; P. Jain and J.P. Ralston, in: Future Directions in Particle and Nuclear Physics at Multi-GeV Hadron Beam Facilities, BNL, March 1993.

[2] X. Ji, Phys. Rev. Lett. 78 (1997) 610; Phys. Rev. D55 (1997) 7114; X. Ji, W. Melnitchouk and X. Song, Phys. Rev. D56 (1997) 5511; X. Ji, J. Phys. G: Nucl. Part. Phys. 24 (1998) 118.

[3] A.V. Radyushkin, Phys. Lett. B380 (1996) 417; Phys. Lett. B385 (1996) 333; Phys. Rev. D56 (1997) 5524.

[4] J. C. Collins, L. Frankfurt, M. Strikman, Phys. Rev. D56 (1997) 2982; J. C. Collins and A Freund, hep-ph/9801262.

[5] M. Diehl et al. Phys. Lett. B411 (1997) 193;

[6] V.Yu. Petrov et al., Phys.Rev. D57 (1997) 4325.

[7] M. Vanderhaegen, P.A.M. Guichon and M. Guidal, Phys. Rev. Lett. 80 (1998) 5064 . 
[8] A.D. Martin and M.G. Ryskin, Phys. Rev. D57 (1998) 6692.

[9] A.V. Radyushkin, hep-ph/9805342.

[10] L. Mankiewicz, G. Piller and T. Weigl, Eur. Phys. J. C5 (1998) 119.

[11] A.V. Belitsky et al., hep-ph/9710427; A.V. Belitsky and A. Schäfer, hepph/9801252; A.V. Belitsky and D. Müller, Phys.Lett. B417 (1998) 129. J. Blümlein, B. Geyer and D. Robaschik, Phys.Lett. B406 (1997) 161.

[12] L. Mankiewicz et al. hep-ph/9712251.

[13] J.P. Ralston and D.E. Soper, Nucl. Phys. B152 (1979) 109; X. Artru and M. Mekhfi, Z. Phys. C45 (1990) 669; J.L. Cortes, B. Pire and J.P. Ralston, Z. Phys. C55 (1992) 409; R.L. Jaffe and X.Ji, Phys. Rev. Lett. 67 (1991) 552; Nucl. Phys. B375 (1992) 527.

[14] J. Soffer, Phys. Rev. Lett. 74 (1995) 1292.

[15] W. Vogelsang, Phys. Rev. D57 (1998) 1886.

[16] C. Bourrely, J. Soffer and O.V. Teryaev, Phys.Lett. B420 (1998) 375.

[17] M. Diehl and T. Gousset, hep-ph/980123.

[18] see, e.g. R.L. Jaffe, hep-ph/9602236.

[19] H. Cornille and A. Martin, Nucl. Phys. B115 (1976) 163.

[20] P. Hoodbhoy and X. Ji, hep-ph/9801369.

[21] C. Bourrely, E. Leader and O.V. Teryaev, hep-ph/9803238; O.V. Teryaev, hep-ph/9803403. 